\title{
"Bio-glues" to Enhance Slipperiness of Mucins: Improved Lubricity and Wear Resistance of Porcine Gastric Mucin (PGM) Layers Assisted by Mucoadhesion with Chitosan
}

Nikogeorgos, Nikolaos; Efler, Petr; Lee, Seunghwan; Kayitmazer, A. Basak

Published in:

Soft Matter

Link to article, DOI:

10.1039/C4SM02021A

Publication date:

2015

Link back to DTU Orbit

Citation $(A P A)$ :

Nikogeorgos, N., Efler, P., Lee, S., \& Kayitmazer, A. B. (2015). "Bio-glues" to Enhance Slipperiness of Mucins: Improved Lubricity and Wear Resistance of Porcine Gastric Mucin (PGM) Layers Assisted by Mucoadhesion with Chitosan. Soft Matter, 11, 489-498. https://doi.org/10.1039/C4SM02021 A

\section{General rights}

Copyright and moral rights for the publications made accessible in the public portal are retained by the authors and/or other copyright owners and it is a condition of accessing publications that users recognise and abide by the legal requirements associated with these rights.

- Users may download and print one copy of any publication from the public portal for the purpose of private study or research.

- You may not further distribute the material or use it for any profit-making activity or commercial gain

- You may freely distribute the URL identifying the publication in the public portal 


\section{Soft Matter}

\section{Accepted Manuscript}

This article can be cited before page numbers have been issued, to do this please use: N. Nikogeorgos,

P. Efler, A. B. Kayitmazer and S. Lee, Soft Matter, 2014, DOI: 10.1039/C4SM02021A.

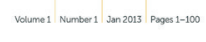

\section{Soft Matter}

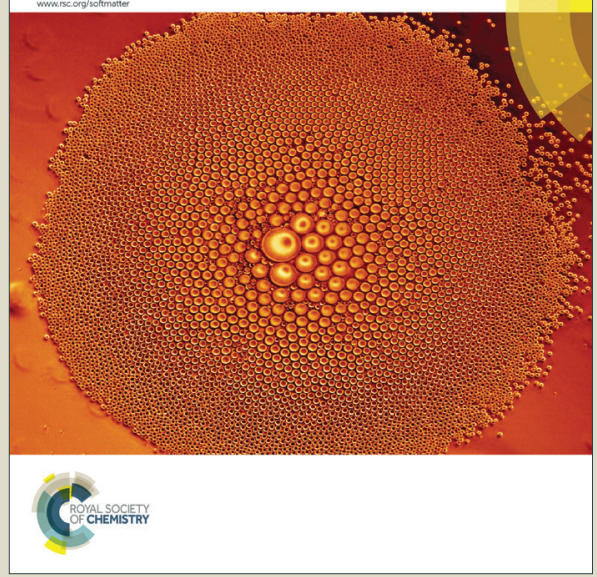

This is an Accepted Manuscript, which has been through the Royal Society of Chemistry peer review process and has been accepted for publication.

Accepted Manuscripts are published online shortly after acceptance, before technical editing, formatting and proof reading. Using this free service, authors can make their results available to the community, in citable form, before we publish the edited article. We will replace this Accepted Manuscript with the edited and formatted Advance Article as soon as it is available.

You can find more information about Accepted Manuscripts in the Information for Authors.

Please note that technical editing may introduce minor changes to the text and/or graphics, which may alter content. The journal's standard Terms \& Conditions and the Ethical guidelines still apply. In no event shall the Royal Society of Chemistry be held responsible for any errors or omissions in this Accepted Manuscript or any consequences arising from the use of any information it contains. 


\title{
"Bio-glues" to Enhance Slipperiness of Mucins: Improved Lubricity and Wear Resistance of Porcine Gastric Mucin (PGM) Layers Assisted by Mucoadhesion with Chitosan
}

\author{
${ }_{5}$ Nikolaos Nikogeorgos ${ }^{a}$, Petr Efler $^{a}$, A. Basak Kayitmazer ${ }^{b}$ and Seunghwan Lee, ${ }^{a}$ \\ Received (in $X X X, X X X)$ Xth $X X X X X X X X X 20 X X$, Accepted $X$ th $X X X X X X X X X 20 X X$ \\ DOI: $10.1039 / b 000000 x$
}

A synergetic lubricating effect between porcine gastric mucin (PGM) and chitosan based on their mucoadhesive interaction is reported at a hydrophobic interface comprised of self-mated

10 polydimethylsiloxane (PDMS) surfaces. In acidic solution $(\mathrm{pH} 3.2)$ and low concentrations $\left(0.1 \mathrm{mg} \mathrm{mL}^{-}\right.$

${ }^{1}$ ), the interaction of PGM with chitosan led to surface recharge and size shrinkage of their aggregates.

This resulted in higher mass adsorption on the PDMS surface with increasing weight ratio of

[chitosan]/[PGM + chitosan] up to 0.50 . While neither PGM nor chitosan exhibited slippery

characteristics, coefficient of friction being close to 1 , their mixture improved considerably the lubricating

15 efficiency (coefficient of friction 0.011 at optimum mixing ratio) and wear resistance of the adsorbed

layers. These findings are explained by the role of chitosan as a physical crosslinker within the adsorbed

PGM layers, resulting in higher cohesion and lower interlayer chain interpenetration and bridging.

\section{Introduction}

${ }_{20}$ Mucins are glycoproteins that consist of a long, linear protein backbone chain, for which the central region is heavily glycosylated, whereas the $\mathrm{C}$ - and $\mathrm{N}$-termini remain largely unglycosylated. ${ }^{1}$ Mucins readily form hydrogels by recruiting a large amount of water, and coat the respiratory, ${ }_{25}$ gastrointestinal, and reproductive tracts, protecting and lubricating the underlying epithelial tissue surfaces. Mucins and mucus gels have thus received particular interest in the areas of drug delivery ${ }^{2-5}$ and biolubrication. ${ }^{6-10}$ In the former case, the major interest lies in the mucoadhesive interaction

30 between mucins and polymers that may be used as drug carriers so as to achieve better control in the delivery and release of drug molecules across the mucus gel on gastrointestinal organs. ${ }^{3}$

An example of a highly studied mucoadhesive polymer is 35 chitosan, a linear cationic polysaccharide that is derived from chitin by partial deacetylation. In addition to its low toxicity, biocompatibility and biodegradability, chitosan is known to display antibiomicrobial, hypoallergenic, wound healing, and film forming properties. ${ }^{2-5}$ Moreover, the amine 40 and hydroxyl groups in chitosan allow regulation of its mucoadhesive properties through chemical derivatization. ${ }^{5}$ Therefore, the mucoadhesive properties of chitosan have been investigated with several techniques such as transmission electron microscopy, ${ }^{11}$ viscosity and turbidity
45 measurements, ${ }^{12-15}$ atomic force microscopy, ${ }^{16,17} \zeta$-potential measurements, ${ }^{14,} 18$ and dynamic light scattering. ${ }^{14}$ The interaction of chitosan with mucins is complicated. It involves electrostatic attraction, hydrogen bonding, and hydrophobic effects, whose relative contributions depend on 50 the exact conditions, such as $\mathrm{pH}$ and the presence of other chemical agents. ${ }^{14,16,19,20}$ At low ionic strength in the $\mathrm{pH}$ range of $2.5-6.5$, the strongest contribution to aggregation comes from the electrostatic interactions. In solution at low chitosan/mucin ratio, chitosan increases mucin's aggregation 55 and a consequent positive synergistic effect in the solution's viscosity is manifested. ${ }^{13-15,18,21,22}$ This was explained by chitosan screening the electrostatic repulsion between the mucin aggregates (mainly due to the negative charge of the sialic and sulfuric acids on the oligosaccharide chains), and 60 by forming polymeric bridges between smaller mucin aggregates. In contrast, as the chitosan/mucin ratio is increased above a critical value, disaggregation takes place due to the chitosan-chitosan electrostatic repulsion, and the size of aggregates decreases again. As such, the interaction ${ }_{65}$ of mucins with chitosan is chiefly concerned with the adhesive properties at the interfaces.

In this study, we demonstrate that this feature can be rather exploited to enhance the slippery nature of mucins, in particular porcine gastric mucin (PGM) at a compliant, 70 hydrophobic interface. By varying the ratio of PGM to chitosan, we have investigated the changes in friction and 
wear resistance properties of PGM-chitosan aggregates at a tribological interface between self-mated poly(dimethylsiloxane) (PDMS) surfaces. The tribological properties were investigated across a large range of contact 5 scale using atomic force microscopy (AFM) and conventional pin-on-disk tribometry. Particular attention was paid to the correlation of the tribological properties with accompanying structural and surface properties of PGMchitosan aggregates at the interface.

\section{${ }_{10}$ Materials and methods}

\section{Mucins and chemicals}

PGM (Type III: partially purified, bound sialic acid $0.5-$ $1.5 \%$ ) and chitosan (Product code: 1001135895, 80\% deacetylated, $\mathrm{MW} \sim 250 \mathrm{kDa}$ ) were purchased from Sigma${ }_{15}$ Aldrich (Denmark Aps, Brøndby, Denmark). All chemicals were laboratory grade, and were also purchased from Sigma Aldrich (Brøndby, Denmark). Solutions of PGM and chitosan were prepared at a concentration of $0.1 \mathrm{mg} \mathrm{mL} \mathrm{m}^{-1}$. The solvent used was a 1:1 (v:v) mixture of phosphate buffer 20 saline $(10 \mathrm{mM}, \mathrm{pH}=7.4$, no extra salts) and $10 \mathrm{mM} \mathrm{HCl}$ solution. The $\mathrm{pH}$ of this solvent mixture was determined as 3.2 (VWR, phenomenal TM). The low $\mathrm{pH}$ and concentrations used in this study were chosen in order to achieve full dissolution of chitosan within a reasonable time 25 in our buffer system. Mixtures of PGM with chitosan were prepared at [chitosan]/[PGM + chitosan] weight ratios of $0.20,0.25,0.33$, and $0.50(\mathrm{w} / \mathrm{w})$. This weight ratio is denoted as [chitosan]/[biopolymer] throughout this manuscript. All mixtures were prepared from the $0.1 \mathrm{mg} \mathrm{mL} \mathrm{m}^{-1}$ solutions of 30 PGM and chitosan so that the total biopolymer concentration in the final mixture remained at $0.1 \mathrm{mg} \mathrm{mL}^{-1}$. All experiments were started exactly 1 hour after preparation of the mixture, unless otherwise stated.

\section{Dialysis purification of PGM}

$35500 \mathrm{mg}$ of PGM were dissolved at a concentration of $10 \mathrm{mg}$ $\mathrm{mL}^{-1}$ in an aqueous solvent of $10 \mathrm{mM}$ Na-phosphate, 150 $\mathrm{mM} \mathrm{NaCl}, 10 \mathrm{mM}$ DTT, and $0.5 \mathrm{mM}$ EDTA, with $\mathrm{pH}$ adjusted at 7.4. Dialysis in CE BioTech tubing with $100 \mathrm{kDa}$ MWCO (Spectrum Labs) against Milli-Q water followed for 40 three days with solvent exchange every $24 \mathrm{~h}$ (total dilution $\left.10^{6}\right)$. Sample was then freeze-dried in Scanvac CoolSafe ${ }^{\mathrm{TM}}$ (Labogene) for three days and saved at $-20{ }^{\circ} \mathrm{C}$ until use.

\section{Zeta potential measurements}

Zeta potential of PGM, chitosan, and their mixtures with 45 varying ratios was characterized with a laser $(633 \mathrm{~nm})$ Doppler electrophoresis instrument (LDE; Zetasizer Nano ZS, Malvern, UK). The $\mathrm{pH}$ of all the solutions was set at 3.2. Disposable cuvettes (model DTS 1070) were used. Three measurements were performed per sample, while each 50 measurement was the average of ten repeats.

\section{Dynamic light scattering (DLS)}

DLS experiments were carried out with a 3D LS Spectrometer (LS Instruments, Switzerland), using the 3D LS Spectrometer v6.3 software. The measurements were 55 performed at room temperature and the scattering angle was fixed at $90^{\circ}$. Size distributions were determined using a CONTIN analysis over at least 10 averaged measurements. ${ }^{23}$ The buffer used was filtered repeatedly $(0.2 \mu \mathrm{m}$ pore size, Sartorius/VWR) prior to dissolution of PGM and chitosan. ${ }_{60}$ All samples were placed in borosilicate cylindrical glass cuvettes (LS Instruments). The mixtures were allowed $1 \mathrm{~h}$ in the cuvettes prior to data acquisition.

\section{Optical waveguide light-mode spectroscopy (OWLS)}

OWLS is based on grating-assisted in-coupling of a He-Ne ${ }_{65}$ laser into a planar waveguide coating (200-nm thick $\mathrm{Si}_{0.25} \mathrm{Ti}_{0.75} \mathrm{O}_{2}$ waveguiding layer on $1 \mathrm{~mm}$ thick AF 45 glass, Microvacuum Ltd, Budapest, Hungary). OWLS experiments were carried out using the OWLS 210 Label-free Biosensor system (Microvacuum Ltd, Budapest, Hungary).

70 Waveguides used in this work were spin-coated at 2500 $\mathrm{rpm}$ for $15 \mathrm{~s}$ with an ultrathin layer $\left(\sim 30 \mathrm{~nm}^{10}\right)$ of polystyrene (Sigma Aldrich, Brøndby, Denmark), $6 \mathrm{mg} \mathrm{mL}^{-1}$ in toluene, and a subsequent ultrathin layer of PDMS. The base and curing agent of a commercial silicone elastomer 75 (Sylgard 184 elastomer kit, Dow Corning, Midland, MI) were dissolved in hexane at a ratio of 10:3 (final concentration, $0.5 \% \mathrm{w} / \mathrm{w})$. The solution was spin-coated onto a waveguide at $2000 \mathrm{rpm}$ for $25 \mathrm{~s}$, and cured in an oven at $70{ }^{\circ} \mathrm{C}$ overnight.

80 The PS/PDMS-coated waveguide was first exposed to PBS until a stable baseline was obtained. A programmable syringe pump (Model 1000-NE, New Era Pump Systems, Inc., NY) was employed to transport buffer solutions through flow-cell containing OWLS waveguide surface. 100 ${ }_{85} \mu \mathrm{L}$ of sample solution was then injected via loading loop. Upon observing an increase in surface adsorption, the pump was stopped for 30 minutes. After rinsing the flow cell with PBS, the adsorbed mass density data were calculated according to de Feijter's equations. ${ }^{24}$ The experiment was 90 repeated three to five times for each sample solution. The refractive index increment $(\mathrm{d} n / \mathrm{d} c)$ value used for the calculation of the adsorption masses was determined for each solution experimentally using an automatic refractometer (Rudolph, J157). The values determined were ${ }_{95} 0.130,0.200,0.165,0.153,0.148,0.144 \mathrm{~cm}^{3} \mathrm{~g}^{-1}$ for PGM, chitosan, and their mixtures [chitosan]/[biopolymer]: 0.50 , $0.33,0.25,0.20 \mathrm{w} / \mathrm{w}$, respectively.

\section{Pin-on-Disk tribometry and tribopair}

Macroscale tribological properties were investigated by pin100 on-disk tribometer (CSM, Peseux, Switzerland). Friction forces between a loaded pin sliding in contact with a disk were measured at controlled rotation speeds of the disk. The friction forces were determined by a strain gauge. The friction forces data obtained in this study were measured 105 over a fixed track (radius: $3 \mathrm{~mm}$ ) and load $(1 \mathrm{~N})$ while 
varying the speed of rotation. An average coefficient of friction $(\mu)$, defined as friction force/load, for each speed was plotted.

The PDMS pins and disks were prepared as follows: the $s$ base fluid and crosslinker of Sylgard 184 elastomer kit (Dow Corning, Midland, MI) were thoroughly mixed at a ratio of 10:1. Air trapped in the mixture was removed by applying a gentle vacuum. Disks were cast in a machined aluminum plate mold with flat wells designed to the dimensions (30 $10 \mathrm{~mm}$ diameter $\times 5 \mathrm{~mm}$ thickness) of the tribometer. For the disks, only the sides exposed to air during curing were used for tribological measurements. The hemispherical pins were molded in a 96 microwell plate (NUNCLON Delta Surface, Roskilde, Denmark). The PDMS mixtures were then cured at $1570{ }^{\circ} \mathrm{C}$ overnight. ${ }^{25}$

\section{Friction force microscopy (FFM)}

FFM experiments were performed with a Digital Instruments Nanoscope III Multimode instrument (Bruker Instruments, Santa Barbara, CA), using V-shaped silicon nitride AFM ${ }_{20}$ probes (model NP, Bruker Instruments, Santa Barbara, CA) with a nominal normal force constant of $0.35 \mathrm{~N} \mathrm{~m}^{-1}$. All measurements were performed in liquids using a liquid cell (Bruker Instruments, Santa Barbara, CA). The normal spring constant of the cantilevers was obtained according to the ${ }_{25}$ generalized Sader method. ${ }^{26-28}$ The normal photodetector sensitivity $\left(\mathrm{nm} \mathrm{V}^{-1}\right)$ was acquired from the slope of the linear part of a force curve at the repulsive regime, obtained on the flat regions of a silica specimen. Calibration of lateral forces was achieved using the 'wedge calibration method'. ${ }^{29} \mathrm{~A}$ 30 silica specimen was used for a horizontal and a sloped $\left(26.5^{\circ}\right.$ angle) surface, prepared using focused ion beam milling. ${ }^{30}$ The radius of curvature of the tips $(33 \pm 5 \mathrm{~nm})$ was characterized with the blind tip reconstruction method using a TGT01 grating (Mikromash). ${ }^{31}$ For friction measurements, 35 the instrument was operated in contact mode with the long cantilever axis perpendicular to the fast scanning direction (1.5 Hz scanning speed), over lines of $1 \mu \mathrm{m}$ length, with the slow scan axis being disabled. Friction forces were determined from 'trace - retrace friction loops' acquired 40 along single lines; the mean signals in opposing directions were subtracted and, subsequently, halved. ${ }^{32,33}$ Three to five different locations were examined on each sample, while two samples and two different cantilevers were used for each system.

${ }_{45}$ Silanized probes were prepared using octadecyltrichlorosilane (OTS, $>90 \%$, Sigma Aldrich). All probes were piranha-cleaned. The piranha solution has very strong oxidizing power, and is extremely dangerous to handle in the laboratory; rubber gloves, safety glasses, and so lab coat are needed for protection. The probes were subsequently rinsed with copious amounts of ultrapure water (Merck Millipore Direct-Q system, Billerica, MA), dried in a flow of nitrogen, and immersed in a mixture solution of 20 $\mathrm{ml}$ cis- and trans-decahydronaphthalene (Merck, >99\%), 1 ${ }_{55} \mathrm{mM}$ in

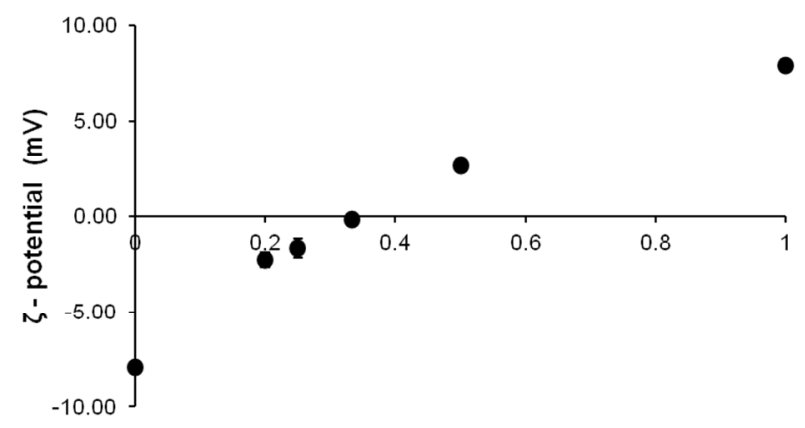

[chitosan] / [biopolymer] weight ratio

Fig. $1 \zeta$-potential data of PGM, chitosan and their mixtures versus the [chitosan]/[biopolymer] weight ratio, where [biopolymer] $=0.1 \mathrm{mg} \mathrm{mL}^{-1}$.

chloroform (Alfa Aesar, HPLC grade, dried and distilled) 60 and OTS for 30 minutes. The probes were subsequently rinsed with cyclohexane (VWR, $>90 \%$ ), then ethanol and dried with $\mathrm{N}_{2}$ flow. The probes were used immediately. The PDMS slabs as substrates for FFM experiments were prepared as described in the previous paragraph. The slabs ${ }_{65}$ were further immersed in toluene for 2 days, then sonicated for $1 \mathrm{~h}$ in acetone and, subsequently, in ethanol for removal of the uncrosslinked chains.

The PDMS slabs were rinsed with ethanol and dried with nitrogen flow. A large drop of the polymeric (PGM, 70 chitosan, and their mixtures) solution was placed onto the PDMS for $30 \mathrm{~min}$ to allow adsorption of the polymeric species. Subsequently, the PDMS slab was gently rinsed with the solvent. The AFM experiments were carried out in the solvent environment.

75

\section{Results and discussion}

Interaction of PGM with chitosan in solution and synergistic adsorption onto hydrophobic surfaces

\section{${ }_{80} \zeta$-potential}

Fig. 1 shows the $\zeta$-potential of the biopolymers in solution as a function of the weight fraction of chitosan with respect to the total weight of biopolymers in solution. $\zeta$-potential measurements have been used previously to examine the 85 mucoadhesion of several biopolymers. ${ }^{18,} 22,34$ The negative value of $-7.9 \pm 0.3 \mathrm{mV}$ for PGM is in good agreement with previous studies. ${ }^{14,22}$ Considering that at $\mathrm{pH}=3.2$ (used in this study) the ionization of the carboxylic groups from sialic acids and peptide core has been suppressed, the major origin 90 of negative charges is considered to be the sulfate groups in PGM. ${ }^{35,36}$ Chitosan, on the other hand, has a positive surface potential due to the protonation of the amino groups at $\mathrm{pH}=$ 3.2. As the weight ratio of chitosan is increased, the surface of the PGM-chitosan aggregates is gradually recharged from 95 negative to positive values, which indicates that chitosan binds onto the mucinous aggregates, thus, changing their $\zeta$ potential towards positive values. The electroneutrality of the aggregates was determined at ca. 0.33 [chitosan]/[biopolymer] weight ratio. 
Table 1 Values of the hydrodynamic radius $R_{H}$ and the relative amount (R.A. \%) as obtained by DLS.

\begin{tabular}{|c|c|c|c|c|c|c|c|c|c|c|c|}
\hline \multicolumn{2}{|c|}{ PGM } & \multicolumn{2}{|c|}{0.2 w/w chit } & \multicolumn{2}{c|}{0.25 w/w chit } & \multicolumn{2}{c|}{0.33 w/w chit } & \multicolumn{3}{c|}{0.5 w/w chit } & \multicolumn{2}{c|}{ chitosan } \\
\hline$R_{H}(\mathrm{~nm})$ & R.A. $\%$ & $\mathrm{R}_{\mathrm{H}}(\mathrm{nm})$ & R.A. $\%$ & $\mathrm{R}_{\mathrm{H}}(\mathrm{nm})$ & R.A. $\%$ & $\mathrm{R}_{\mathrm{H}}(\mathrm{nm})$ & R.A. $\%$ & $\mathrm{R}_{\mathrm{H}}(\mathrm{nm})$ & R.A. $\%$ & $\mathrm{R}_{\mathrm{H}}(\mathrm{nm})$ & R.A. $\%$ \\
\hline $165 \pm 70$ & 10 & $109 \pm 33$ & 9.7 & $117 \pm 23$ & 21.3 & $82 \pm 26$ & 8.5 & - & - & $219 \pm 50$ & 98.4 \\
\hline $383 \pm 140$ & 89 & $343 \pm 75$ & 90 & $323 \pm 116$ & 78.4 & $301 \pm 138$ & 91 & $176 \pm 15$ & 99.1 & - & - \\
\hline
\end{tabular}
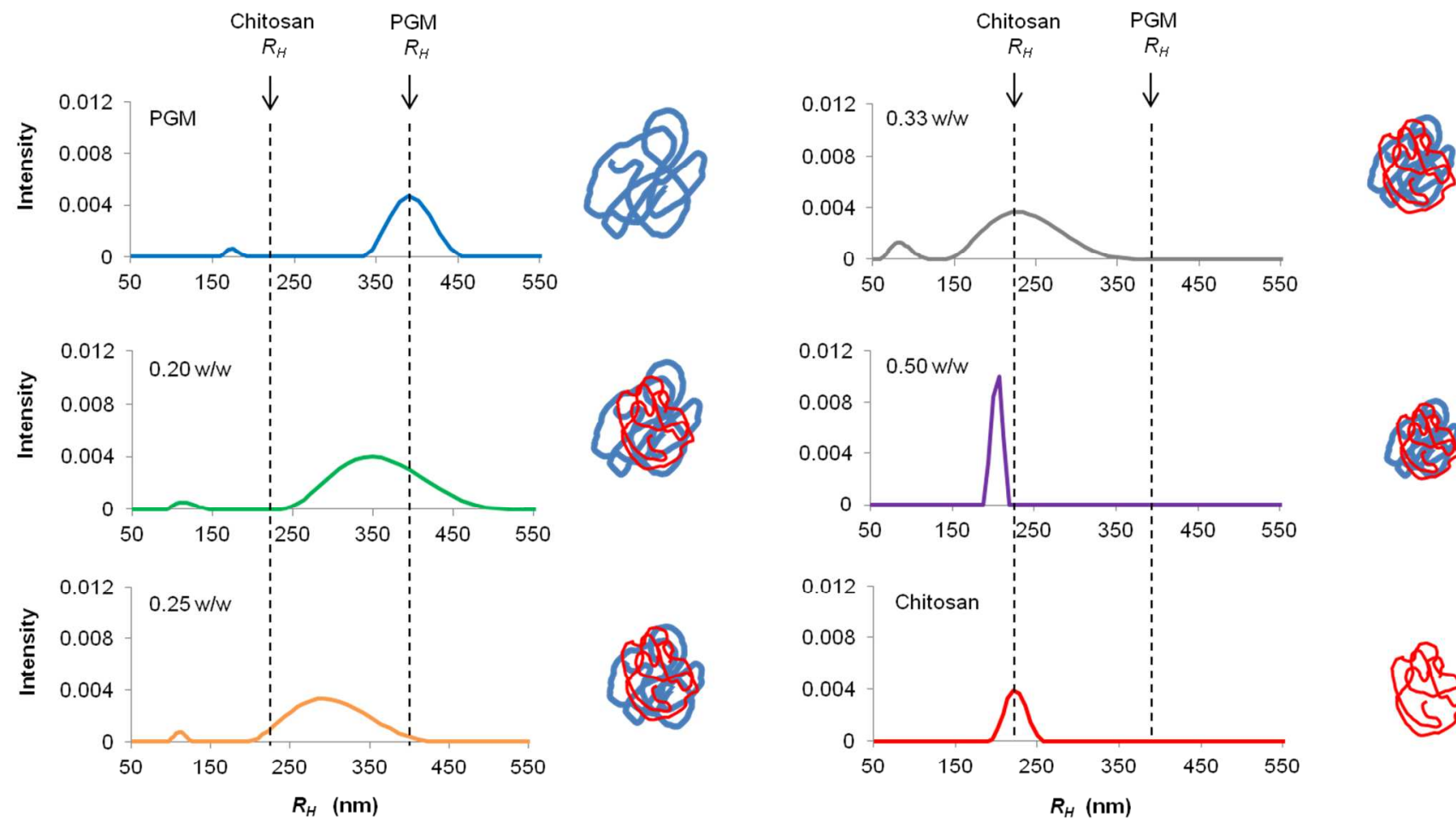

5 Fig. 2 Intensity weighted particle size distributions of $0.1 \mathrm{mg} \mathrm{mL}^{-1} \mathrm{PGM}$, chitosan, and their mixtures (denoted according to the [chitosan]/[biopolymer] weight ratio) obtained by DLS. The vertical lines represent the position of the average hydrodynamic radius of chitosan and PGM as obtained from their DLS spectra. On the right side of each graph there is an illustration of the shrinkage resulting from the interaction of PGM with chitosan.

\section{Dynamic light Scattering (DLS)}

Fig. 2 shows the intensity weighted particle size distributions 10 of the solutions as examined by DLS, and Table 1 shows the values of the derived hydrodynamic radius, $R_{H}$, and their relative amount. As has been previously observed, ${ }^{14,} 22$ PGM is characterized by a bimodal size distribution with populations at $165 \pm 70 \mathrm{~nm}$ and $383 \pm 140 \mathrm{~nm}$. The large 15 standard deviation reflects the polydispersity of the aggregates' size. In contrast, chitosan has one population at $219 \pm 50 \mathrm{~nm}$. In the mixtures, as the chitosan weight ratio is increased, both populations gradually shift towards lower values until the 0.50 weight ratio of [chitosan]/[biopolymer] 20 is reached where there is only one peak at $176 \pm 15 \mathrm{~nm}$.

Fig. 3 shows the change in $R_{H}$ of the largest population with respect to the [chitosan]/[biopolymer] weight ratio. A clear trend can be seen that as the weight ratio of chitosan in the mixture is increased the size of the PGM-chitosan 25 aggregates is decreased. This means that the interaction of chitosan with PGM results in a shrinkage of the PGM- chitosan aggregates. To further investigate the effect of $\mathrm{PGM} /$ chitosan interaction in solution, circular dichroism spectra were acquired from all samples (data in Supporting 30 Information). All samples exhibited random coil conformation in solution, which indicates that the secondary structure of the aggregates is not affected appreciably by the PGM-chitosan interaction in solution. As shown by the $\zeta$ potential measurements (Fig. 1), DLS and CD spectroscopy, ${ }_{35}$ PGM binds with chitosan in solution, leading to aggregates of increased surface potential, decreased size, and yet sustained random coil conformation. Sogias et $\mathrm{al}^{14}$ showed that the interaction of PGM with chitosan at low $\mathrm{pH}$ is chiefly due to electrostatic attraction, although contributions 40 from hydrogen bonding, van der Waals, and hydrophobic interactions still contribute weakly. We propose that the chitosan molecules bind onto PGM mainly through electrostatic attraction between the $\mathrm{NH}_{4}{ }^{+}$and $\mathrm{SO}_{4}{ }^{-}$groups, thus shifting the surface charge towards positive values and 45 lowering the intramolecular electrostatic repulsion of the PGM molecules, hence, collapsing their size. 


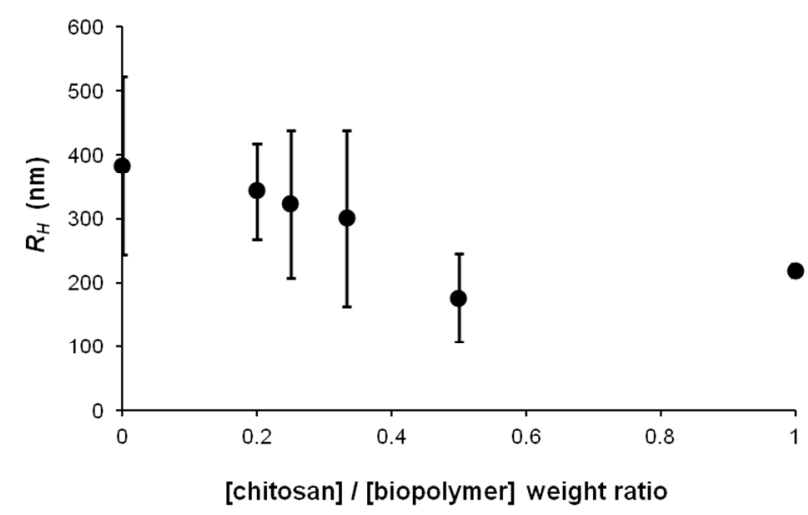

Fig. 3 Hydrodynamic radius, $R_{H}$, of the solutions examined as obtained by DLS versus the [chitosan]/[biopolymer] weight ratio, where [biopolymer] $=0.1 \mathrm{mg} \mathrm{mL}^{-1}$.

This finding appears to contradict previous studies on the interaction of chitosan with mucins in solution, which have shown that at these weight ratios of chitosan the opposite trend should be observed. ${ }^{11}$ 14, 15, 18, 21, 22 However, in those 10 studies, the concentrations of both chitosan and mucinous solutions were considerably greater $\left(\geq 1 \mathrm{mg} \mathrm{mL}^{-1}\right)$ than those in the present study, $0.05-0.1 \mathrm{mg} \mathrm{mL}^{-1}$. Similar discrepancy due to concentration effects has appeared between past studies. $^{12,13,15}$ Hassan and Gallo ${ }^{15}$ employed viscometry to 15 evaluate the mucoadhesion of chitosan with commercial PGM, and found a positive synergistic effect of mucins with chitosan, where the viscosity of the mixture solution was greater than the sum of the viscosities of the neat chitosan and mucinous solutions. On the other hand, Rossi et al ${ }^{12}$ 20 studied the rheological properties of the mixtures of chitosan with bovine submaxillary mucins (BSM) or PGM, and found a minimum viscosity in the mixtures rather than in the neat chitosan or mucinous solutions for all cases, manifesting a negative synergistic effect. This discrepancy was resolved by ${ }_{25}$ Rossi et $a l^{13}$ where it was shown that when chitosan solutions are of concentrations lower than its characteristic entanglement concentration (cec), a negative rheological synergistic effect is observed upon interaction with PGM, whereas at concentrations higher than cec, a positive 30 synergistic effect is manifested. In the present study, the concentrations of both chitosan and PGM vary in the different solutions from 0.002 to $0.01 \mathrm{w} / \mathrm{v} \%$, which is clearly within the range where a negative rheological synergistic effect should be expected. Indeed, a negative size 35 synergistic effect is shown by the DLS data where the aggregates' hydrodynamic size in chitosan/PGM mixtures is lower than that of the sum of their sizes when alone in solution. Moreover, the fact that $\zeta$-potential was determined to be closest to neutrality at $0.33 \mathrm{w} / \mathrm{w}$ ratio, while the 40 minimum aggregates' size was exhibited by the $0.50 \mathrm{w} / \mathrm{w}$ mixture, indicates that even though electrostatic interactions are the primary driving force for inter-diffusion

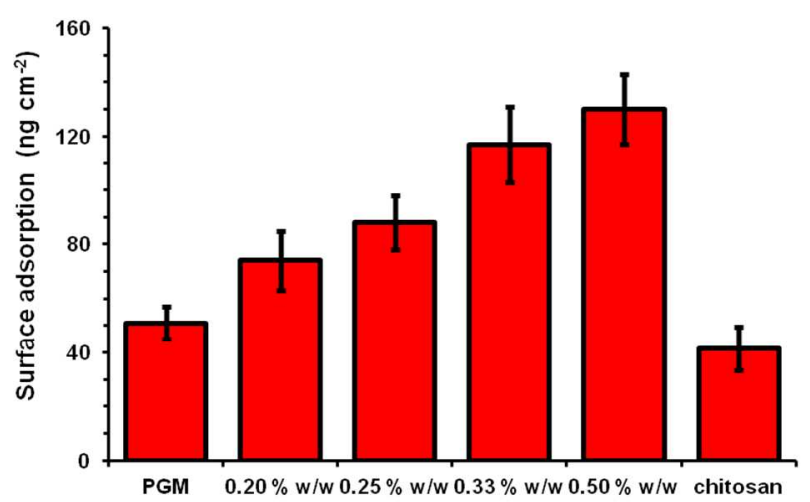

Fig. 4 Surface adsorbed masses on PDMS spin-coated waveguides of 0.1 $45 \mathrm{mg} \mathrm{mL}^{-1}$ PGM, chitosan, and their mixtures as characterized by optical waveguide lightmode spectroscopy (OWLS). Adsorption was allowed to occur for 30 minutes. Mixtures denoted according to the [chitosan]/[biopolymer] weight ratio, where [biopolymer] $=0.1 \mathrm{mg} \mathrm{mL}^{-1}$.

of PGM and chitosan in low pH solutions, other interactions 50 such as hydrogen bonding, Van der Waals and hydrophobic ones, also play a secondary but non-negligible role in the resulting aggregates' size. ${ }^{14}$

\section{Optical waveguide light spectroscopy (OWLS)}

${ }_{55}$ As can be seen in Fig. 4, neither chitosan nor PGM do adsorb in large amounts onto PDMS. One reason for this is the short time given (30 min) for adsorption, which was not enough for saturation. This is because OWLS was employed in this study to characterize the adsorption behavior of the ${ }_{60}$ polymers under tribological stress in pin-on-disk tribometry configuration. Under such a configuration, desorption/(re)adsorption of the biopolymers onto the PDMS is repeatedly established due to the cyclic tribostress, and the surface adsorption is far from equilibrium. ${ }^{37}$ Moreover, the ${ }_{65}$ low adsorbed masses for PGM and chitosan are expected considering the low concentrations used $\left(0.1 \mathrm{mg} \mathrm{mL}^{-1}\right)$ and the polyelectrolyte characteristics of both biopolymers. But, it is notable that the adsorption of PGM is slightly higher than that of chitosan. This is presumably because, unlike 70 chitosan, PGM possesses distinct surface anchoring groups, namely unglycosylated hydrophobic patches in the $\mathrm{C}$ - and N-termini.

As the weight fraction of chitosan in the solution increases, so does the adsorbed mass. This can be explained 75 primarily by the decreasing surface charge of the PGMchitosan aggregates with increasing amount of chitosan up to a weight ratio of 0.50 [chitosan]/[biopolymer] (Fig. 1). A lower surface charge results in weaker electrostatic repulsion between the aggregates on the nonpolar PDMS surface, 80 facilitating higher mass adsorption. However, this is not necessarily the case for the mixture at 0.50 [chitosan]/[biopolymer] w/w, since the lowest surface charge is observed for the ratio of 0.33 [chitosan]/[biopolymer] $\mathrm{w} / \mathrm{w},-0.2 \mathrm{mV}$, (Fig. 1). Thus, it is argued that the size of ${ }_{85}$ PGM-chitosan aggregates plays as a second factor in the 
surface adsorption properties. Previous studies have shown

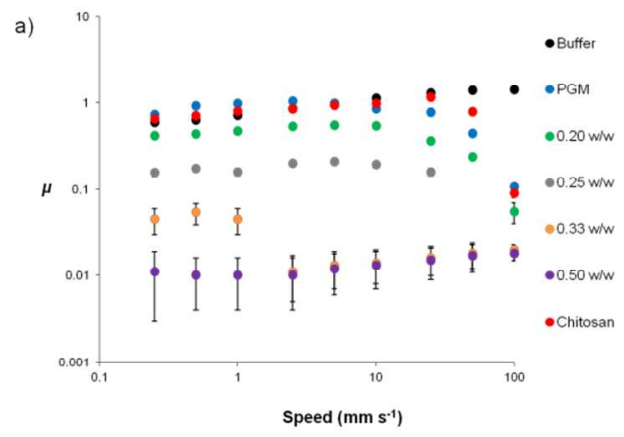

that the adsorption of mucinous species onto hydrophobic

Fig. 5 Pin-on-disk data of the PDMS/PDMS interface for PGM, chitosan, and their mixtures obtained at $1 \mathrm{~N}$ load. Mixtures denoted according to the 5 [chitosan]/[biopolymer] weight ratio, where [biopolymer] $=0.1 \mathrm{mg} \mathrm{mL}^{-1}$. a) Data acquired in the polymeric solutions $\left(\left[\right.\right.$ biopolymer] $\left.=0.1 \mathrm{mg} \mathrm{mL}^{-1}\right)$ with varying speed, b) data obtained from the polymeric coatings at $5 \mathrm{~mm} \mathrm{~s}^{-1}$ in buffer solution; prepared after 30 min adsorption of the polymers onto PDMS and rinsing with buffer, c) data of [chitosan]/[biopolymer] at 0.50 weight ratio at $5 \mathrm{~mm} \mathrm{~s}^{-1}$ speed in different ways of mixing and sequential addition of the biopolymers.

surfaces is initially diffusion-controlled. ${ }^{38,39}$ Since the size 10 of the aggregates is decreased by the addition of chitosan (Fig. 2), their diffusion rates increase accordingly. Thus, smaller aggregates can adsorb faster onto the surface. Adsorption profiles as a function of time (data not shown) indicate that saturation was not reached within the ${ }_{15}$ experiment's time scale (ca. 30 minutes) for all of the samples. Thus, faster adsorption kinetics can readily lead to larger amount of adsorbed mass, too. The adsorption mechanism of the PGM-chitosan aggregates onto PDMS should not be substantially different from that of PGM alone, 20 as the interaction between PGM-chitosan occurs essentially through glycan-chitosan interaction, whereas $\mathrm{C}$ - and $\mathrm{N}$ terminal hydrophobic patches of PGM may act as anchoring units, ${ }^{1,} 38$ leading to the so called 'loop-and-tail' conformation. ${ }^{40}$ Regardless of the net surface charges as a 25 result of interaction with chitosan, the glycosylated regions of PGM are still hydrophilic and would not display a favorable interaction with the PDMS surface.

\section{Lubricating properties of PGM/chitosan layers between hydrophobic surfaces}

30

\section{Macroscale}

The macroscale lubricating properties of PGM, chitosan, and their mixtures were investigated using a pin-on-disk (PoD) tribometer for a PDMS-PDMS tribopair. The mixtures were 35 allowed to stabilize for $1 \mathrm{~h}$ prior to the experiments. Additionally, all samples were given $30 \mathrm{~min}$ to adsorb on the pin and disk before the start of sliding contacts in the solutions, unless otherwise stated. Fig. 5a shows the friction coefficient $(\mu)$ of the various samples as a function of speed. 40 Both PGM and chitosan display very poor lubricating capabilities. Only at the highest speed $\left(100 \mathrm{~mm} \mathrm{~s}^{-1}\right), \mu$ is 0.11 and 0.09 , respectively, whereas with decreasing speed, $\mu$ rapidly increases. At speeds lower than $25 \mathrm{~mm} \mathrm{~s}^{-1}, \mu$ exhibits no lubricating effect and its values are equal to those of the 45 sliding contacts of PDMS/PDMS in the buffer. In a previous study, ${ }^{10}$ PGM has been shown to adsorb in greater amounts and lubricate efficiently the self-mated PDMS in acidic conditions. Thus, poor lubricating properties of PGM in this study are attributed to the much lower concentration and the 50 consequent lower adsorbed masses. As the chitosan/PGM weight ratio is increased, the $\mu$ values are gradually decreased. For example at $0.25 \mathrm{~mm} \mathrm{~s}^{-1}, \mu$ is $0.415,0.157$, $0.045,0.011$ for the $0.2,0.25,0.33,0.5 \mathrm{w} / \mathrm{w}$ [chitosan]/[biopolymer] weight ratio, respectively. It can ${ }_{55}$ also be seen that in all mixtures, the "threshold speed" (at which low $\mu$ values in high-speed regime rapidly increase with lowering speed) gradually decreases with increasing chitosan weight ratio. This threshold speed can be an indication that the lubricating mechanism may switch from 60 boundary lubrication (low-speed regime) to fluid-film lubrication (high-speed regime).

Fig. $5 b$ shows the friction coefficient $(\mu)$ of the various samples as a function of the number of revolutions. It should be noted that in these experiments the tribopair was rinsed ${ }_{65}$ with buffer solution after the samples were given $30 \mathrm{~min}$ to adsorb on the pin and disk, and thus no excess biopolymers were present in the buffer solution. Both PGM and chitosan appear to be removed immediately from within the contact area by the tribostress. However, PGM is removed more 70 slowly than chitosan possibly due to the relatively stronger binding of its hydrophobic parts at the $\mathrm{N}$ - and C-termini onto the PDMS. As the weight ratio of chitosan is increased in the mixture, the number of revolutions for which friction is maintained low also increases. This means that the cohesion 75 of the lubricant layer also increases with the weight ratio of 


\section{Cite this: DOI: $10.1039 / \mathrm{c0xx00000x}$}

\section{www.rsc.org/xxxxxx \\ ARTICLE TYPE}

a)

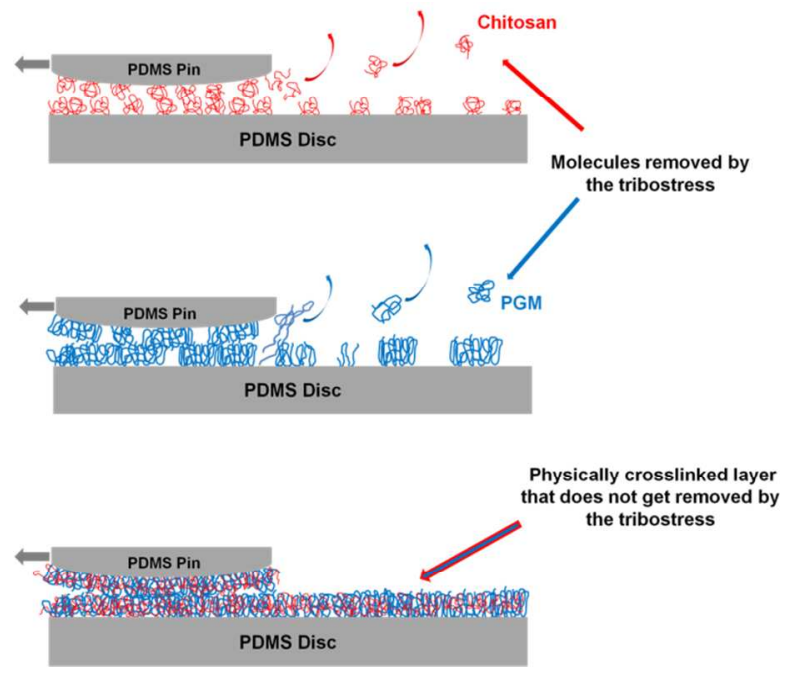

b)
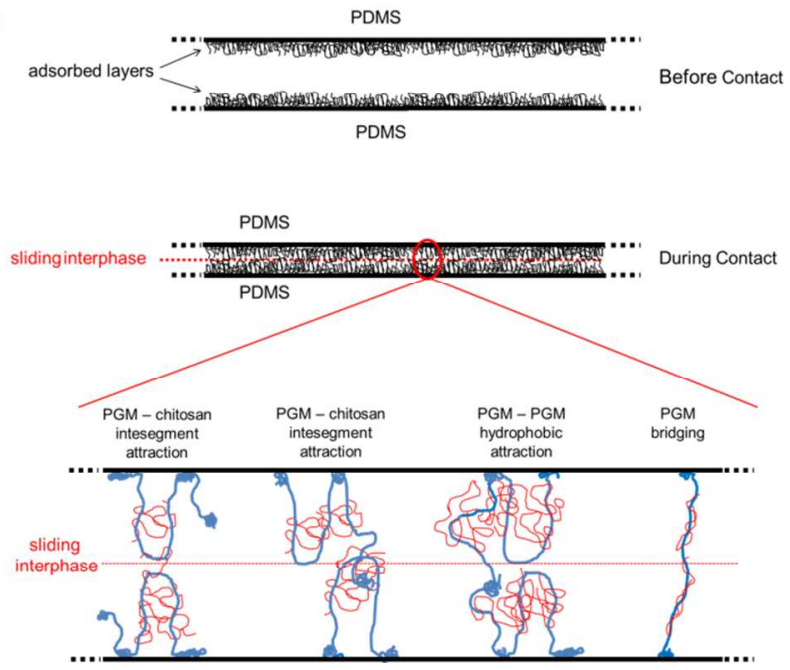

Fig. 6 a) Illustration of the tribostress-induced removal of the adsorbed layers from the PDMS surface and their resistance to it in the presence of chitosan, b) illustration of the chain interpenetration interactions between two PGM / chitosan layers.

5 chitosan, leading to a layer of considerably better wear resistance. Namely, the highest wear resistance to the tribostress is observed from the PGM-chitosan mixture layer of the 0.50 [chitosan]/[biopolymer] weight ratio which is not removed for a sliding distance of more than $5 \mathrm{~m}$.

10 Past studies with BSM at the mica/silica interface have shown that the friction between mucin layers is increased upon addition of chitosan. ${ }^{17}$ However, there are two major differences between that study and the present study. Firstly, in that study, ${ }^{17}$ negatively charged surfaces were employed 15 whereas neutral ones were employed in the present study. Secondly, Pettersson et al. ${ }^{17}$ added mucin and chitosan sequentially, i.e., chitosan was added after the mucinous layer was already formed and the addition of chitosan led to a collapse of the mucinous layer and a considerable increase 20 in friction. ${ }^{17}$ On the other hand, our study so far involved pre-mixing of the two biopolymers. For this reason, we also tested sequential adsorption with our system (PDMS/PDMS) by employing the 0.50 [chitosan]/[biopolymer] mixture to examine whether premixing of PGM with chitosan is 25 important in the lubrication and wear properties of the final layer (Fig. 5c). For the systems where chitosan or PGM was added first (black and purple circles, respectively) upon addition of the second polymer a sudden drop is seen in $\mu$, but not to an extent close to the premixed case (Fig. 5, $\mu=$ 30 0.011). Furthermore, $\mu$ starts increasing immediately after this initial decrease. Alternatively, PGM was allowed to adsorb first for $30 \mathrm{~min}$ before starting the experiment, and once $\mu$ increased to the buffer value, equal volume of chitosan was injected, and the system was allowed for $1 \mathrm{~h}$
35 before sliding contacts between the PDMS surfaces restarted (blue circles). When sliding contacts restarted, $\mu$ dropped to the value comparable to those of the premixed solutions, but quickly increased to a value of $\mu=0.8$. The same sequential injection experiments were repeated, yet 40 without pre-tribological contacts with the first biopolymer alone, but the $\mu$ values were again gradually increased (data not shown).

We tried two different ways of premixing experiments. Firstly, the samples were premixed for $1 \mathrm{~h}$ in solution, 45 placed upon the PDMS tribopair for $30 \mathrm{~min}$, and then friction forces were recorded (filled red circles). Secondly, equal volumes of PGM and chitosan were injected at the same time on PDMS tribopair and allowed to adsorb for 30 min (open yellow circles) and $1 \mathrm{~h}$ (open green circles). In all 50 cases, the value of $\mu$ remained below 0.02 for at least 1000 revolutions (where the experiment was stopped). In particular, for the first premixing case, the system maintained its lubricity for more than 3000 revolutions corresponding to more than $50 \mathrm{~m}$ of sliding distance. ${ }_{55}$ Considering that in the buffer (Fig. 5b) wear of the adsorbed layer takes place after about 300 revolutions, the lubricant layer in polymer solution exhibits superior wear resistance, suggesting that the presence of a reservoir of PGM-chitosan aggregates in the solution assists the maintenance of ${ }_{60}$ lubricating layer under consistent tribological stress. ${ }^{37}$ This series of data (Fig. 5c) shows that sequential adsorption of PGM and chitosan does not result in effective lubrication, regardless of the order of adsorption between the two biopolymers. But, for effective lubrication, premixing 
outside the tribopair is not necessary either. Instead, coadsorption of the two species onto the tribopair surface appears to be the key requirement for effective lubrication.
According to Fig. 5a and b, as the chitosan weight ratio ${ }_{5}$ is increased, both the anti-friction properties and the wear

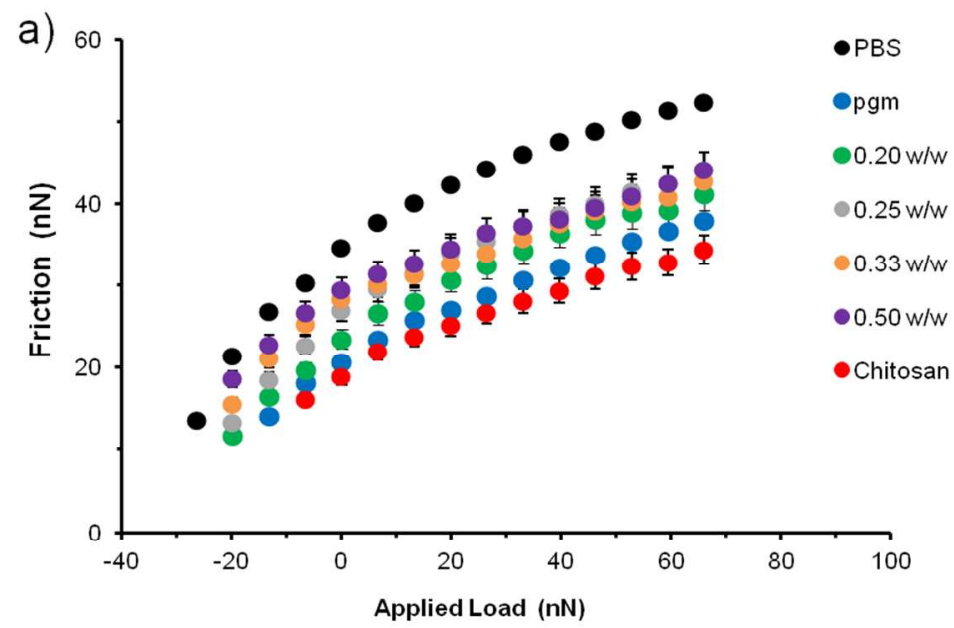

b)
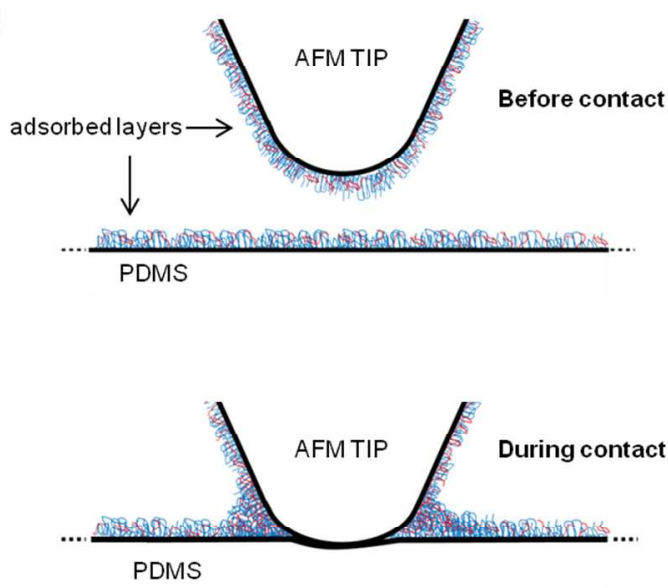

Fig. 7 a) Friction force microscopy data of $0.1 \mathrm{mg} \mathrm{mL}^{-1}$ PGM, chitosan and their mixtures obtained from the contact between OTS silanized probes and PDMS slabs in the buffer solution (mixtures denoted according to the [chitosan] / [biopolymer] weight ratio, where [biopolymer] $=0.1 \mathrm{mg} \mathrm{mL}^{-1}$.), b) illustration of the plowing of the probe through the crosslinked network during FFM measurements.

10

resistance to the tribostress are considerably improved. Similarly with the case where cations form intermolecular links between the negatively charged parts of mucins, ${ }^{41,} 42$ chitosan may act as a physical crosslinker within the PGM 15 layer based on its polycationic character at low pH. It is well established that both physical and chemical crosslinking within a lubricant layer improve its load bearing and wear resistance capabilities, whether this layer is chemi- or physisorbed on the surface. ${ }^{43-52}$ Thus, it is reasonable to 20 assume that chitosan physically crosslinks the adsorbed mucinous layer through chain entanglement and formation of electrostatic bridges between the glycosylated parts of PGM, hence, leading to a layer of much higher cohesion and wear resistance (Fig. 6a). This is in agreement with Fig. 5b, 25 where at high [chitosan]/[biopolymer] weight ratios no increase in friction force is observed for several hundred rotations even without excess polymers in bulk solution. The crosslinking between PGM and chitosan does not necessarily enhance the direct interaction of PGM or chitosan with the 30 PDMS substrates, but intensifies the lateral interaction across all the biopolymers on the surface, and thus stabilizes the lubricating film. Insufficient wear resistance of the PGM-chitosan layer by sequential adsorption (Fig. 5c) suggests that there might be an optimal arrangement between ${ }_{35}$ PGM and chitosan on the surface in order to form a strongly crosslinked layer that displays excellent anti-wear properties. In other words, when the PDMS surface is pre-occupied with a PGM or chitosan layer, the interaction with the incoming second biopolymer may not lead to a sufficiently stable 40 crosslinking that could withstand the applied tribostress.

Previous studies have shown that crosslinking of the adsorbed polymer layer is more often related to increased friction forces, despite the improvement in wear resistance. ${ }^{43,44,46,47,49,50,52}$ However, the crosslinked PGM45 chitosan layers in this study show both lower friction forces and improved wear resistance. According to Fig. 5a, in the case of $0.25,0.33,0.50 \mathrm{w} / \mathrm{w}$ [chitosan]/[biopolymer] weight ratios, the adsorbed layers do not get removed within the number of laps used (20-50) to determine $\mu$ and their values 50 are $0.157,0.045,0.011$ at $0.25 \mathrm{~mm} / \mathrm{s}$ (Fig. 5a), respectively, i.e. they decrease with increasing chitosan ratio. This can be explained by the different inter-diffusion ability due to intersegment attraction and bridging between the opposing surfaces. $^{2,3,8,9,17,53-56}$ For the specific polymer systems in 55 this study, hydrophobic interactions of the unglycosylated blocks of mucins with those of the opposing mucinous layers, or with the opposing PDMS surface, as well as electrostatic interactions between mucin/chitosan chains in opposing layers can reinforce chain interpenetration events 60 between the opposing layers (Fig. 6b). The likelihood of chain interpenetration is dependent on the chitosan weight ratio for several reasons. Firstly, as the [chitosan]/[biopolymer] weight fraction is increased, the surface adsorption mass is also increased, leading to higher 65 chain density on and, consequently between the surfaces. Higher chain density allows less space for interpenetration and bridging to occur due to excluded volume effects. ${ }^{57}$ Secondly, chitosan's ability to physically crosslink the adsorbed layer results in higher lateral entanglement density, 70 where the chains are more restricted within the layer and less free to escape it and inter-diffuse across the sliding interface. $^{49-53,} 55, \quad 58 \quad$ Finally, at $0.50 \quad \mathrm{w} / \mathrm{w}$ [chitosan]/[biopolymer] weight ratio, the electrostatic double layer repulsion between the opposing layers according to ${ }_{75}$ Fig. 1 should be the highest for which the likelihood of 
removal of the layer due to the tribostress is the least (Fig. $5 b)$.

At last, it should not be neglected that the lubricant layer formed at the weight ratio of 0.50 ([chitosan]/[biopolymer]) 5 does also exhibit "self-healing" ability. ${ }^{37}$ Although its wear resistance is maintained up to ca. 300 revolutions of the pin in the buffer (Fig. 5b), when there is a reservoir of the PGMchitosan aggregates in the solution, no wear of the film is observed for more than 3500 revolutions (Fig. 5c). This is an 10 important additional property of the film that can be expected from physically crosslinked films.

\section{Nanoscale}

15 The tribological properties of PGM, chitosan and their mixtures were also investigated by FFM using OTSfunctionalized probes and a PDMS slab. All the measurements were performed in buffer solution by rinsing the tribopair and leaving a layer of the adsorbed biopolymers 20 on the surfaces. As shown in Fig. 7a, similarly with macroscale contacts, all biopolymer samples show lower friction in comparison to the reference buffer, primarily due to lower adhesion forces. However, the nanoscale tribological properties of the samples are in clear contrast to 25 their macroscale properties. Firstly, both PGM and chitosan exhibit the lowest friction forces, whereas all the mixtures show higher frictional properties with the 0.50 [chitosan]/[biopolymer] weight ratio displaying the highest friction. Secondly, their frictional differences are much less 30 pronounced at the scale studied by FFM. Integrated probes have a very low radius $(\sim 30 \mathrm{~nm})$, and thus can penetrate the biopolymer layer under loading conditions. ${ }^{59}$ Hence, the friction experienced by the tip is due to its interaction with the substrate, and also due to the resistance applied to it by

35 the adsorbed layer as it plows through it. This is illustrated in Figure $7 \mathrm{~b}$.

At the nanoscale the tip and the PDMS are in mechanical contact due to the loading force, and, consequently, the tip has to plow through the adsorbed layer (Fig. 7b). A higher 40 mass density, such as the film obtained from the [chitosan]/[PGM] at the ratio of 0.33 or $0.50 \mathrm{w} / \mathrm{w}$, means higher number of chains and bonds encountered by the tip per unit length of sliding. Therefore, higher energy is dissipated per unit length of sliding by the tip in ${ }_{45}$ breaking/disrupting these bonds (chitosan-PGM electrostatic bridges, PGM-PDMS hydrophobic bonds, chain entanglements) as it plows its way through the layer. Moreover, a higher degree of entanglement within the layer may result in a stiffer layer, ${ }^{44,45}$ which could also explain a 50 higher force needed by the tip to slide through it. The films formed from neat PGM or chitosan show the lowest friction forces, due to the lack of crosslinked bridges between the molecules on the same surface.

\section{${ }_{55}$ Conclusions}

In this study, we have demonstrated that mucoadhesion properties between PGM and chitosan can be exploited to enhance the lubricating properties of PGM at a compliant, hydrophobic interface composed of self-mated PDMS 60 surfaces. The interaction of PGM with chitosan was examined in aqueous solutions at low concentrations and $\mathrm{pH}$ (3.2). The surface of the PGM becomes gradually recharged from negative to positive potential upon mixing with increasing portion of chitosan, leading to a negative ${ }_{65}$ synergistic effect in their aggregates' size. Their interaction, however, did not affect the secondary structure of PGM or PGM-chitosan aggregates appreciably, but remained to display random coil structure in all cases. These interactions are accounted for mainly by the electrostatic attraction 70 between the oppositely charged sulfate groups on the glycosylated blocks of PGM and the amino groups of chitosan at this $\mathrm{pH}$ (3.2), although other interactions also appear to contribute. ${ }^{14}$ The adsorbed mass onto the PDMS surface increased as the weight ratio [chitosan]/[PGM] 75 increased up to 0.50 , due to faster diffusion and weaker electrostatic repulsion between the aggregates on the nonpolar PDMS surface. The macroscale lubricating efficiency and wear resistance of PGM were also found to improve with increasing amount of chitosan in the mixture.

${ }_{80}$ The best lubricity and wear resistance of the aggregates were exhibited at the 0.50 [chitosan]/[biopolymer] weight ratio with a coefficient of friction of 0.011 , i.e. two orders of magnitude lower than that of chitosan or PGM alone. This was explained by chitosan acting as a physical crosslinker 85 within the adsorbed PGM layer, thus, increasing its cohesion and allowing less chain interpenetration/bridging across the sliding interface. However, sequential adsorption of chitosan and PGM (regardless of the order) did not lead to similarly improved lubricity. Instead, only when the two polymers are 90 allowed to co-adsorb simultaneously onto the PDMS, the low friction is maintained (e.g. for more than 3500 laps on the same sliding track). At nanoscale tribological contacts studied by FFM, however, an opposite trend was observed in terms of relative friction forces between PGM, chitosan, and 95 their aggregates. Both chitosan and PGM showed lower friction than their mixtures, due to the higher contact pressures at the nanoscale contacts, where the predominant friction mechanism is plowing of the AFM probe through the polymer layer on the surface.

\section{${ }_{100}$ Acknowledgements}

The European Research Council is acknowledged for their financial support (Funding scheme, ERC Starting Grant 2010, Project number 261152) for this work. The COST Action CM1102 is also acknowledged for financial support. Dr. Maher ${ }_{105}$ Abou Hachem and Prof. Birte Svensson (Dept. of Systems Biology, Technical University of Denmark) are acknowledged for collaboration with PGM purification. Mr. Zoltan Imre Balogh (Center for Electromicroscopy, DTU) is appreciated for preparing the silicon wedge for AFM lateral forces calibration. Mr. Ioannis 110 Papadimitriou is acknowledged for providing soft wear programs 
(using Wolfram Mathematica) that assisted the calibration of the AFM normal and lateral forces.

\section{Notes and references}

${ }^{a}$ Department of Mechanical Engineering, Technical University of 5 Denmark, DK-2800 Kgs. Lyngby, Denmark. Fax: +45459362 13; Tel: +4545252193; E-mail:seele@mek.dtu.dk

${ }^{b}$ Bogazici University, Department of Chemistry, TR-34342 Istanbul, Turkey.

$\dagger$ Electronic Supplementary Information (ESI) available: [Circular 10 dichroism data]. See DOI: 10.1039/b000000x/

1. R. Bansil, E. Stanley and J. T. LaMont, Annu. Rev. Physiol., 1995, 57, 635-657.

2. N. A. Peppas and J. J. Sahlin, Biomaterials, 1996, 17, 1553-1561.

15 3. N. A. Peppas and Y. Huang, Adv. Drug Delivery Rev., 2004, 56, 1675-1687.

4. J. D. Smart, Adv. Drug Delivery Rev., 2005, 57, 1556-1568.

5. V. V. Khutoryanskiy, Macromol. Biosci., 2011, 11, 748-764.

6. G. Cassin, E. Heinricha and H. A. Spikes, Tribol. Lett., 2001, 11, 95 20 102 .

7. E. Perez and J. E. Proust, J. Colloid Interface Sci., 1987, 118, 182191.

8. M. Malmsten, E. Blomberg, P. Claesson, I. Carlstedt and I. Ljusegren, J. Colloid Interface Sci., 1992, 151, 579-590.

25 9. N. M. Harvey, G. E. Yakubov, J. R. Stokes and J. Klein, Biomacromolecules 2011, 12, 1041-1050.

10. S. Lee, M. M. ller, K. Rezwan and N. D. Spencer, Langmuir, 2005, 21, 8344-8353.

11. I. Fiebrig, S. E. Harding, A. J. Rowe, S. C. Hyman and S. S. Davis, Carbohydr. Polym., 1995, 28, 239-244.

12. S. Rossi, F. Ferrari, M. C. Bonferoni and C. Caramella, Eur. J. Pharm. Sci., 2000, 10, 251-257.

13. S. Rossi, F. Ferrari, M. C. Bonferoni and C. Caramella, Eur. J. Pharm. Sci., 2001, 12, 479-485.

35 14. I. A. Sogias, A. C. Williams and V. V. Khutoryanskiy, Biomacromolecules, 2008, 9, 1837-1842.

15. E. Hassan and J. Gallo, J. Pharm. Res., 1990, 7, 491-495.

16. M. P. Deacon, S. McGurk, C. J. Roberts, P. M. Williams, S. J. Tendler, M. C. Davies, S. S. Davis and S. E. Harding, Biochem. J., 2000, 348, 557-563.

17. T. Pettersson and A. Dedinaite, J. Colloid Interface Sci., 2008, 324, 246-256.

18. H. Takeuchi, J. Thongborisute, Y. Matsui, H. Sugihara, H. Yamamoto and Y. Kawashima, Adv. Drug Delivery Rev., 2005, 57, 1583-1594.

19. M. P. Deacon, S. S. Davis, R. J. White, H. Nordman, I. Carlstedt, N. Errington, A. J. Rowe and S. E. Harding, Carbohydr. Polym., 1999, 38, $235-238$

20. D. Snyman, J. H. Hamman and A. F. Kotze, Drug Dev. Ind. Pharm., 2003, 29, 61-69.

21. N. Thirawong, R. A. Kennedy and P. Sriamornsak, Carbohydr. Polym., 2008, 71, 170-179.

22. N. A. Fefelova, Z. S. Nurkeeva, G. A. Mun and V. V. Khutoryanskiy, Int. J. Pharm., 2007, 339, 25-32.

55 23. S. W. Provencher, Comput. Phys. Commun., 1982, 27, 229-242.

24. J. J. Ramsden, J. Stat. Phys., 1993, 73, 853-877.

25. T. Røn, I. Javakhishvili, K. Jankova, S. Hvilsted and S. Lee, Langmuir, 2013, 29, 7782-7792.

26. J. E. Sader, J. Pacifico, C. P. Green and P. Mulvaney, J. Appl. Phys., 2005, 97, 124903-124903.

27. J. E. Sader, J. A. Sanelli, B. D. Adamson, J. P. Monty, X. Wei, S. A. Crawford, J. R. Friend, I. Marusic, P. Mulvaney and E. J. Bieske, Rev. Sci. Instrum., 2012, 83, 103705.

28. J. W. M. Chon, P. Mulvaney and J. E. Sader, J. Appl. Phys., 2000, 87, 3978-3988.

29. M. Varenberg, I. Etsion and G. Halperin, Rev. Sci. Instrum., 2003, 74, 3362-3367.
30. E. Tocha, H. Schonherr and G. J. Vancso, Langmuir, 2006, 22, 23402350.

70 31. A. Yacoot and L. Koenders, J. Phys. D: Appl. Phys. 41, 2008, 41, 103001.

32. S. Graftstrom, M. Neitzert, T. Hagen, J. Ackerman, R. Probst and M. Wortge, Nanotechnology, 1993, 4, 143-151.

33. R. Overney and E. Meyer, MRS Bull., 1993, 18, 26-34.

75 34. J. Song, D. Tranchida and G. J. Vancso, Macromolecules, 2008, 41, 6757-6762.

35. N. G. Karlsson, I. Carlstedt, J. R. Davies, A. Herrmann, H. Nordman and G. C. Hansson, Biochem. J., 1997, 326, 903-910.

36. T. Sandberg, M. K. Ott, J. Carlsson, A. Feiler and K. D. Caldwell, $J$. Biomed. Mater. Res. Part A, 2008, 93A, 773-785.

37. S. Lee, M. Müller, R. Heeb, S. Zürcher, S. Tosatti, M. Heinrich, F. Amstad, S. Pechmann and N. D. Spencer, Tribol. Lett., 2006, 24, 217-223.

38. L. Shi and K. D. Caldwell, J. Colloid Interface Sci., 2000, 224, 372 381 .

39. L. Lindh, P. O. Glantz, I. Carlstedt, C. Wickstro"m and T. Arnebrant, Colloids and Surfaces B: Biointerfaces 2002, 25, 139-146.

40. A. A. Feiler, A. Sahlholm, T. Sandberg and K. D. Caldwell, J. Colloid Interface Sci., 2007, 315, 475-481.

90 41. T. Pettersson, Z. Feldoto, P. Claesson and A. Dedinaite, in Surf. Interfaces: Progr. Colloid Polym. Sci., Springer Berlin Heidelberg, 2008, vol. 134, pp. 1-10.

42. Y. Su, Y. Xu, L. Yang, S. Weng, R. D. Soloway, D. Wang and J. Wu, J. Mol. Struct., 2009, 920, 8-13.

95 43. M. Benz, N. Chen and J. Israelachvili, J. Biomed. Mater. Res. Part A, 2004, 71A, 6-15.

44. N. Kampf, U. Raviv and J. Klein, Macromolecules, 2004, 37, 11341142.

45. G. Francius, J. Hemmerlé, J. Ohayon, P. Schaaf, J.-C. Voegel, C. Picart and B. Senger, Microsc. Res. Tech., 2006, 69, 84-92.

46. C. Drummond, P. Richetti, J. RodrÃguez-Hern $\tilde{A}_{i}$ ndez and S. Lecommandoux, The J. Adh., 2007, 83, 431-448.

47. D. Gourdon, Q. Lin, E. Oroudjev, H. Hansma, Y. Golan, S. Arad and J. Israelachvili, Langmuir, 2007, 24, 1534-1540.

105 48. K. Chawla, S. Lee, B. P. Lee, J. L. Dalsin, P. B. Messersmith and N. D. Spencer, J. Biomed. Mater. Res. Part A, 2009, 90A, 742-749.

49. J. M. Lagleize, P. Richetti and C. Drummond, Tribol. Lett., 2010, 39, 31-38.

50. J. Yu, X. Banquy, G. W. Greene, D. D. Lowrey and J. N. Israelachvili, Langmuir, 2012, 28, 2244-2250.

51. M. Kobayashi, M. Terada and A. Takahara, Faraday Discuss., 2012, 156, 403-412.

52. S. Giasson, J.-M. Lagleize, J. RodrÃguez-HernÃ $\tilde{j n d e z}$ and C. Drummond, Langmuir, 2013, 29, 12936-12949.

115 53. N. A. Peppas and P. A. Buri, J. Controlled Release, 1985, 2, $257-$ 275.

54. M. Berry, T. J. McMaster, A. P. Corfield and M. J. Miles, Biomacromolecules 2001, 2, 498-503.

55. E. Jabbari and N. A. Peppas, Polymer, 1995, 36, 575-586.

120 56. J. M. Coles, D. P. Chang and S. Zauscher, Curr. Opin. Colloid Interface Sci., 2010, 15, 406-416.

57. H. Ji, D. Hone, P. A. Pincus and G. Rossi, Macromolecules, 1990, 23, 698-707.

58. N. Chen, N. Maeda, M. Tirrell and J. Israelachvili, Macromolecules, 2005, 38, 3491-3503.

59. N. Nikogeorgos, J. B. Madsen and S. Lee, Colloids and Surfaces B: Biointerfaces, 2014, 122, 760-766. 


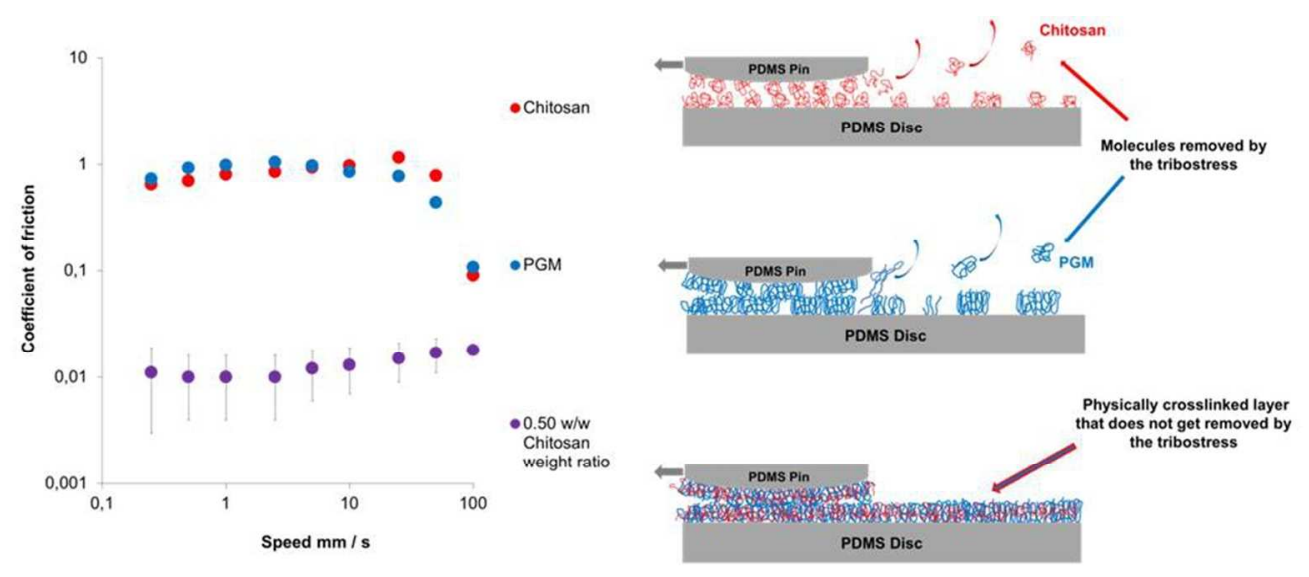

$170 \times 73 \mathrm{~mm}(150 \times 150 \mathrm{DPI})$ 